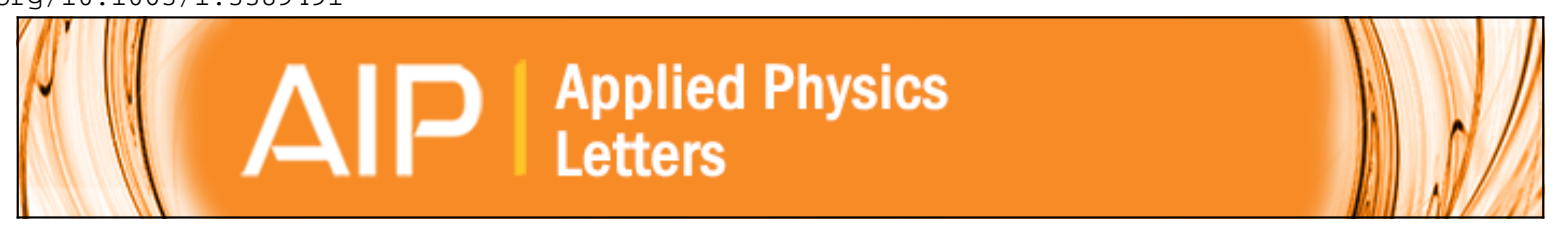

Frequency-dependent stability of parallel-plate electrostatic actuators in conductive fluids

T. L. Sounart, H. V. Panchawagh, and R. L. Mahajan

Citation: Applied Physics Letters 96, 203505 (2010); doi: 10.1063/1.3389491

View online: $\mathrm{http} / / / \mathrm{dx}$. doi.org/10.1063/1.3389491

View Table of Contents: http://scitation.aip.org/content/aip/journal/apl/96/20?ver=pdfcov

Published by the AIP Publishing

Over 700 papers \&
presentations on

multiphysics simulation www nows 


\title{
Frequency-dependent stability of parallel-plate electrostatic actuators in conductive fluids
}

\author{
T. L. Sounart, ${ }^{1, a)}$ H. V. Panchawagh, ${ }^{2, b)}$ and R. L. Mahajan ${ }^{3}$ \\ ${ }^{1}$ Sandia National Laboratories, Albuquerque, New Mexico 87185, USA \\ ${ }^{2}$ Department of Mechanical Engineering, University of Colorado at Boulder, Boulder, \\ Colorado 80309, USA \\ ${ }^{3}$ Department of Mechanical Engineering, Department of Engineering Science and Mechanics, Institute \\ for Critical Technology and Applied Science, Virginia Tech, Blacksburg, Virginia 24060, USA
}

(Received 23 December 2008; accepted 21 March 2010; published online 19 May 2010)

\begin{abstract}
We present an electromechanical stability analysis of passivated parallel-plate electrostatic actuators in conductive dielectric media and show that the pull-in instability can be eliminated by tuning the applied frequency below a design-dependent stability limit. A partial instability region is also obtained, where the actuator jumps from the pull-in displacement to another stable position within the gap. The results predict that the stability limit is always greater than the critical actuation frequency, and therefore any device that is feasible to actuate in a conductive fluid can be operated with stability over the full range of motion. (c) 2010 American Institute of Physics.
\end{abstract}

[doi:10.1063/1.3389491]

Electrostatic actuation is of fundamental importance in applied physics and widely used in microelectromechanical systems (MEMS). The stable range of motion of the common parallel-plate configuration is limited to $1 / 3$ of the initial electrode gap by a well-known electromechanical instability, called the pull-in instability. ${ }^{1}$ Recently it has been shown that the stable actuation range can be extended by adding an electret layer to the gap ${ }^{2}$ or by operating in a high-dielectric fluid medium with relatively thick low-dielectric solid insulating layers on the electrodes. ${ }^{3,4}$ Electrostatic actuation in highdielectric media requires ac drive signals at frequencies $f$ at least on the order of the critical actuation frequency $f_{\mathrm{c}}$ to prevent electrode polarization and electrolysis, ${ }^{5-7}$ but offers the advantage of lower actuation voltages, and is particularly important to microfluidic and bioMEMS development where it is desirable to integrate actuators into aqueous solutions and other conductive fluids. ${ }^{8-12}$ For simplicity and to minimize the applied potential required for actuation, most applications of electrostatic actuators in conductive media have been operated in the high frequency limit, viz., $f \gg f_{\mathrm{c}}=\left(1 / 2 \pi R_{1} C_{\mathrm{d}}\right)_{x=0}$, where $x$ is displacement, $R_{\mathrm{l}}$ is the resistance of the fluid between the electrodes, and $C_{\mathrm{d}}$ is the capacitance of the both passivation layers on the electrodes. The analysis of Legrand $e t a l .{ }^{4}$ that described device design constraints for pull-in suppression in dielectric liquids applies only in this high-frequency limit, where actuation is independent of frequency. In this letter, we present a general stability analysis of parallel-plate electrostatic actuators in conductive fluids, which reveals frequency-dependent stability phenomena for $\Omega=f / f_{\mathrm{c}}=O(1)$, such as frequencydependent pull-in suppression and partial instability where the actuator jumps from the pull-in displacement to another stable position within the gap.

\footnotetext{
${ }^{\text {a) }}$ Author to whom correspondence should be addressed. Present address: Intel Corporation, 4500 Dobson Rd, MS: OC2-210, Chandler, AZ 85248, USA. Tel.: 480-715-2549. Electronic mail: thomas.1.sounart@ intel.com.

${ }^{b)}$ Present address: Eastman Kodak Company. Electronic mail: hrishikesh.panchawagh@kodak.com.
}

Consider the parallel-plate electrostatic actuator in Fig. 1 , driven by a harmonic ac signal, with a spring constant $k$, initial gap $g$, and solid dielectric layers (e.g., from a native or grown oxide) of thickness $t_{\mathrm{d}}$ on each electrode. The impedance of the electrical double-layer in the gap is typically much lower than that of the passivation layers on microactuator devices, and thus can be neglected. ${ }^{6}$ Thus for negligible resistance of the bulk electrode relative to the fluid in the gap, the actuator can be accurately represented by the $R C$ circuit also in Fig. 1. Conservation of energy dictates that the mean electrostatic force $F_{\mathrm{e}}$ is given by ${ }^{7}$

$$
F_{\mathrm{e}}=\frac{1}{2} V_{1 \_ \text {rms }}^{2} \frac{d C_{1}(x)}{d x}=\frac{1}{2} \frac{\varepsilon_{0} \varepsilon_{1} A V_{1 \_ \text {rms }}^{2}}{(g-x)^{2}},
$$

where $C_{1}, \varepsilon_{1}$, and $V_{1 \text { rms }}$ are, respectively, the capacitance, relative dielectric constant, and root mean square potential difference across the fluid in the gap, $\varepsilon_{0}$ is the permittivity of free space, and $A$ is the electrode area.

Now consider an applied sinusoidal drive signal $V(t)=V_{\mathrm{a}} \sin (2 \pi f t)$. The solution to Kirchoff's laws gives ${ }^{7}$

$$
\frac{V_{1 \_ \text {rms }}}{V_{\mathrm{rms}}}=\sqrt{\frac{\Omega^{2}(1-X)^{2}}{1+\Omega^{2}(1+\rho-X)^{2}}},
$$

where $X=x / g$ and $\rho=\left(C_{1} / C_{\mathrm{d}}\right)_{x=0}$ is the undisplaced capacitance ratio. Since $f_{\mathrm{c}}$ and the damping factor in liquids are

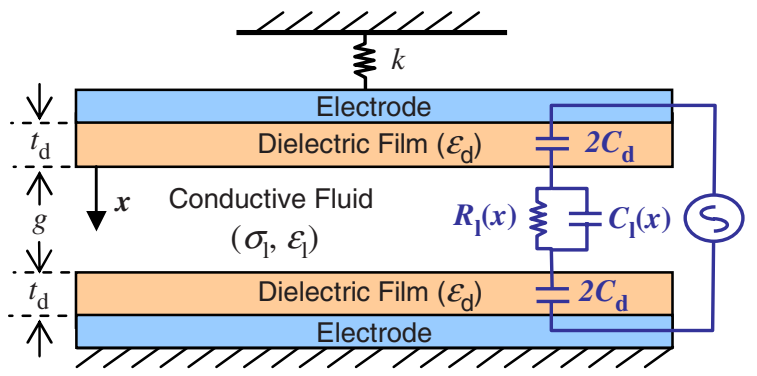

FIG. 1. (Color online) Schematic of a parallel-plate electrostatic actuator in a conductive dielectric medium. $C_{\mathrm{l}}=\varepsilon_{\mathrm{l}} A /(g-x), C_{\mathrm{d}}=\varepsilon_{\mathrm{d}} A / t_{\mathrm{d}}$. 

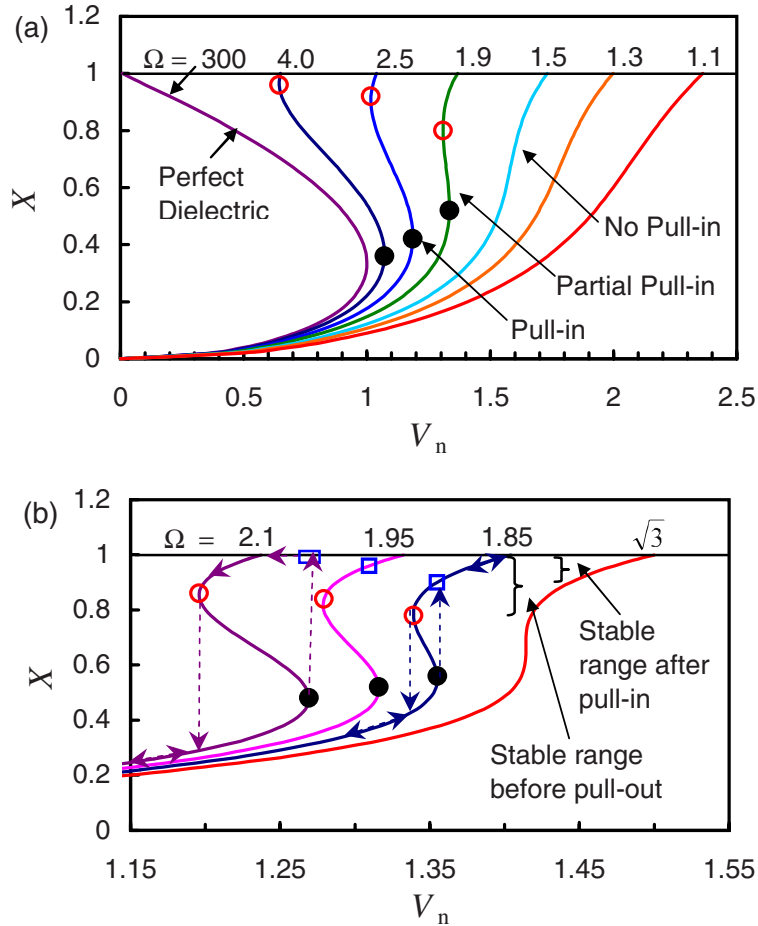

FIG. 2. (Color online) Displacement vs applied potential for $\rho \ll 1$ and selected values of $\Omega$. (a) Wide $\Omega$ range showing curve shape transition. (b) Stability limit region showing stable range of actuator after pull-in and before pull-out. Arrows show actuator paths assuming no structural limitations (e.g., dimples).

usually sufficiently high that actuator dynamic response is negligible, ${ }^{6,7}$ the static displacement of the parallel-plate actuator is obtained by substituting Eq. (2) in Eq. (1), and balancing the electrostatic force with the mechanical restoring force $k x$, viz.,

$$
V_{\mathrm{n}}=\frac{V_{\mathrm{rms}}}{V_{\mathrm{p}}^{\lim }}=\frac{3}{2} \sqrt{3 X\left[\frac{1+\Omega^{2}(1+\rho-X)^{2}}{\Omega^{2}}\right]},
$$

where the rms amplitude of the applied voltage $V_{\text {rms }}$ has been normalized by the rms pull-in voltage $V_{\mathrm{p}}$ in the high $\Omega$ and low $\rho$ limit; $V_{\mathrm{p}}^{\lim }=\sqrt{4 \mathrm{~kg}^{3} /\left(27 A \varepsilon_{1} \varepsilon_{0}\right)}$. The scaled pull-in displacement $X_{\mathrm{p}}$ is determined by taking the derivative of Eq. (3) with respect to $X$ and equating it to zero to obtain the minimum real root

$$
X_{\mathrm{p}}=\frac{(1+\rho)}{3}\left[2-\sqrt{1-\frac{3}{(1+\rho)^{2} \Omega^{2}}}\right] .
$$

The voltage-displacement characteristics for $\rho \ll 1$ (e.g., a typical actuator with a native oxide) are plotted for selected frequencies in Fig. 2. As the frequency is reduced, the shape of the voltage-displacement curve changes from the familiar shape at high frequencies until there are two roots for $d V_{\mathrm{n}} / d X=0$ that are less than 1 . The lowest of the two roots (solid dot) is $X_{\mathrm{p}}$, and inspection of the voltage-displacement curves shows that the higher root (open circle) marks the onset of another stability range of positive $d X / d V_{\mathrm{n}}$. When the two roots are less than 1 , but $V_{\mathrm{n}}(X=1)<V_{\mathrm{p}}$, this second stable range has no impact on actuator pull-in stability, but it defines a second stable range during pull-out $[\Omega=2.1$ in Fig. 2(b)]. For negligible stopper structure height, the actuator jumps to $X=1$ after pull-in Subsequently, as the actuation

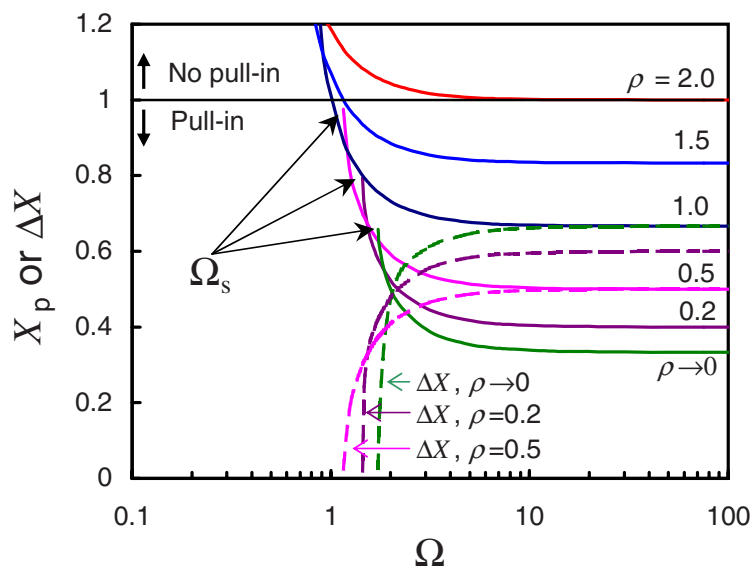

FIG. 3. (Color online) Frequency response of $X_{\mathrm{p}}$ and $\Delta X$. At $\Omega=\Omega_{\mathrm{s}}$, $\Delta X=0$ and $X_{\mathrm{p}}$ curves terminate.

potential is lowered, the actuator displacement is continuously reduced, following the voltage-displacement curve in the second stable range until reaching the highest root, which defines $X_{\mathrm{po}}$ [open circle, Fig. 2(b)]. At this point, the actuator becomes unstable and "pulls out" to the stable region at lower displacements. As the frequency is further reduced, $X_{\mathrm{p}}$ increases while $X_{\mathrm{po}}$ decreases, and over a narrow frequency range where $V_{\mathrm{n}}(X=1)>V_{\mathrm{p}}$ just below the stability limit $\Omega_{\mathrm{s}}$, the actuator does not pull-in all the way to $X=1[\Omega=1.85$ in Fig. 2(b)]. It reaches another stable point (open square) where displacement can be increased with increasing voltage. As $\Omega$ is lowered toward $\Omega_{\mathrm{s}}$ in this partial pull-in region, the distance the actuator jumps from $X_{\mathrm{p}}$ to the next stable position diminishes continuously to zero at $\Omega_{\mathrm{s}}=\sqrt{3}$; for $\Omega<\Omega_{\text {s }}$, there are no real roots, i.e., there is no pull-in instability because $d X / d V_{\mathrm{n}}>0$ for all $X$.

The frequency response of $X_{\mathrm{p}}$ defined by Eq. (4) is shown in Fig. 3 for a range of values of $\rho$. The capacitance ratio $\rho$ is controlled primarily by the thickness of the dielectric layers relative to the electrode gap $\left(\rho \propto t_{\mathrm{d}} / g \sim 10^{-4}\right.$ to 1$)$ because of greater range of control than for the ratio of the dielectric constants $\left(\rho \propto \varepsilon_{1} / \varepsilon_{\mathrm{d}} \sim 2\right.$ to 80$)$. For $\Omega \gg 1, X_{\mathrm{p}}$ is the same as in a perfect dielectric, i.e. $(1+\rho) / 3$. For $\Omega \sim O(1), X_{\mathrm{p}}$ increases sharply with decreasing frequency until reaching the stability limit $\Omega_{\mathrm{s}}$ where $X_{\mathrm{p}}$ ceases to exist. The distance the actuator jumps during pull-in $\Delta X$ diminishes sharply from $1-X_{\mathrm{p}}$ for $\Omega \gg 1$, to zero at $\Omega_{\mathrm{s}}$. $X_{\mathrm{p}}$ also increases with increasing $\rho$, and pull-in is eliminated for all frequencies if $\rho \geq 2$ because $X_{\mathrm{p}}>1$, which is consistent with previous results for pull-in suppression in the high frequency limit $(\Omega \gg 1){ }^{4}$ The more general analysis here shows that $\rho \geq 2$ (which typically requires dielectric layers within an order of magnitude of the gap thickness) is not required to suppress the pull-in instability, which can be eliminated for any $\rho$ simply by tuning the applied frequency. The suppression of pull-in is a result of shifting an increasing percentage of the potential drop across the solid dielectric layers with decreasing $\Omega$ and increasing $\rho$. As the actuator is displaced, the electric field partitions even more into these layers, mitigating the nonlinear increase in electrostatic force with displacement dictated by Eq. (1).

A stability diagram for parallel-plate electrostatic actuators in Fig. 4 shows the regions of stability, instability, and partial stability as a function of $\rho$ and $\Omega$. The stability region 
is divided into two regions; one where stability exists from $X_{\mathrm{p}}>1$, and the other where stability exists due to $\Omega<\Omega_{\mathrm{s}}$, where $X_{\mathrm{p}}$ does not exist. $\Omega_{\mathrm{s}}$ is calculated from $\Delta X=0$ to be simply $\Omega_{\mathrm{s}}=\sqrt{3} /(1+\rho)$. The region where $X_{\mathrm{p}}>1$ is bound by curve $\Omega_{\mathrm{s}}^{\prime}=1 / \sqrt{\rho(2-\rho)}$, derived by equating the higher critical point of $V_{\mathrm{n}}$ to 1 . The $\Omega_{\mathrm{s}}^{\prime}$ curve is tangent to the $\Omega_{\mathrm{s}}$ curve at $\Omega=2 \sqrt{3} / 3, \rho=1 / 2$ (T in Fig. 4). For $\rho>1 / 2, \Omega_{\mathrm{s}}^{\prime}$ defines the stability limit. For $\rho<1 / 2, \Omega_{\mathrm{s}}$ defines the stability limit. The minimum of the $\Omega_{\mathrm{s}}^{\prime}$ curve at $\Omega=1, \rho=1$ (M in Fig. 4) defines the lowest frequency required to eliminate the pull-in instability for any parallel-plate actuator. Thus all actuators are stable at applied frequencies less than or equal to the critical frequency, and actuators will remain stable at increasingly higher frequencies as $\rho$ deviates farther from $\rho=1$. In the limit $\rho \ll 1$ (i.e., $t_{\mathrm{d}} / g \ll 1$ ), pull-in is eliminated for $\Omega<\sqrt{3}$. From a practical standpoint, it is an important result that the stability limit exists universally at $\Omega \geq 1$ because $\Omega \geq O(1)$ is typically required to actuate any device. ${ }^{6,7}$

Below the tangent point $\mathrm{T}, \Omega_{\mathrm{s}}$ and $\Omega_{\mathrm{s}}^{\prime}$ bound the small partial stability region where two stable ranges exist within the gap. The narrow region of partial pull-in displacement within the partial stability range is defined by $V_{\mathrm{n}}(X=1)>V_{\mathrm{p}}$, and thus bound per Eq. (3) by $\Omega_{\mathrm{p}}=2 / \sqrt{1+4 \rho}$ and $\Omega_{\mathrm{s}}$. Although the partial stability region applies to a limited parameter space, this unusual behavior exists for all actuators with $\rho<0.5$, which includes typical native silicon devices in water $(\rho \sim 0.04)$, which are predicted to experience partial pull-in for $\Omega$ between 1.67 and 1.86. This is more than a $10 \%$ change in frequency, and thus easily controlled with common waveform generators.

It is always desirable to minimize the applied voltage required for actuation. Applied voltage requirements increase with increasing $\rho$ and decreasing $\Omega$, but stability requires decreasing $\Omega$ if $\rho<2$. Thus there is an optimum $\rho$ that minimizes the applied voltage requirement for stable actuation over the full range. The minimum voltage $V_{\mathrm{ns}}$ required for stable actuation to a displacement $X$ is given by substitution of $\Omega_{\mathrm{s}}$ and $\Omega_{\mathrm{s}}^{\prime}$ in Eq. (3) to yield:

$$
V_{\mathrm{ns}}= \begin{cases}\frac{3}{2} \sqrt{3 X\left[(1-X)^{2}+2 \rho(2-X)\right]}, & \rho>1 / 2, \\ \frac{3}{2} \sqrt{X\left[(1+\rho)^{2}+3(1+\rho-X)^{2}\right]}, & \rho<1 / 2 .\end{cases}
$$

The inset in Fig. 4 shows that $V_{\mathrm{ns}}$ increases monotonically with $\rho$ for all $X$, and is thus minimized by minimizing $\rho$ and using frequency controlled stabilization (Ref. 13). As $\rho$ approaches zero, such as for devices passivated with a native oxide, the applied voltage required for stable actuation to $X=1$ is only $50 \%$ higher than $V_{\mathrm{p}}^{\text {lim }}$. To achieve stability in the high frequency limit with $\rho>2,{ }^{4}$ the applied voltage must be over five times $V_{\mathrm{p}}^{\lim }$.

The electromechanical stability analysis presented here has profound implications for parallel-plate electrostatic actuators. Stabilization via frequency control has many advantages over stabilization in the high frequency limit with thick passivation layers $(\rho>2)$. With frequency control, any device can be stabilized over the full range of motion, much lower voltages are required, and pull-in suppression can be switched on or off through operational control. The use of an electret can improve stability in air, but large extensions of the stable range require most of the gap to be filled with the electret layer, ${ }^{2}$ which may not be practical in microsystems,

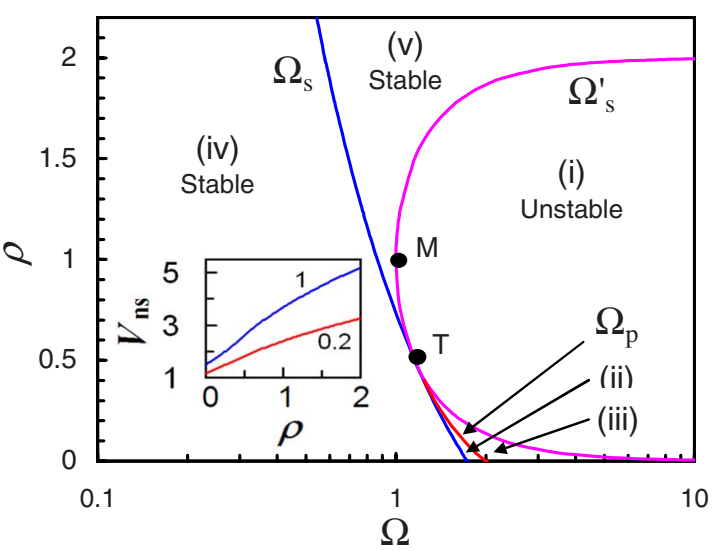

FIG. 4. (Color online) Parallel-plate electrostatic actuator stability diagram in conductive fluids. Five distinct regions: (i) Fully unstable (unstable for all $X>X_{\mathrm{p}}$ ), (ii) partial pull-in instability (stable range exists after pull-in), (iii) partial pull-out instability (stable range exists before pull-out), (iv) stable because $X_{\mathrm{p}}$ does not exist, i.e., no roots of $d V_{\mathrm{n}} / d X=0$, and (v) stable because $X_{\mathrm{p}}>1$. The inset shows the minimum voltage required to actuate a device to $X=1$ and 0.2 without instability (Ref. 13).

and the voltage requirements are higher. Actuator stability in conducting fluids is delineated by two nondimensional parameters, $\Omega$ and $\rho$. Thus, the stability limit $\Omega_{\mathrm{s}}$ is a function only of the capacitance ratio $\rho$, which scales primarily on $t_{\mathrm{d}}$, but also increases with increasing $\varepsilon_{1}$, decreasing $\varepsilon_{\mathrm{d}}$, and decreasing $g$. For thick passivation layers, there is no stability limit, but the applied voltage requirement is maximum; for thin passivation layers, the stability limit is greater than the actuation frequency $\left(\Omega_{\mathrm{s}}=\sqrt{3}\right.$ for $\left.\rho \ll 1\right)$ and the applied voltage requirement is minimized. The partial stability region obtained for $\rho<0.5$ is unique to frequency-controlled stabilization, and is of fundamental interest in understanding electrostatic actuator behavior in conductive fluids. And since typical devices have $\rho<0.5$, the ability to control a variable step within the gap might also be harnessed in future microsystem applications.

Sandia National Laboratories is a multi program laboratory operated by Sandia Corporation, a Lockheed Martin Co., for the Department of Energy under Contract No. DEAC04-94AL85000.

${ }^{1}$ S. D. Senturia, Microsystem Design (Kluwer, Boston, 2001), pp. 130-137.

${ }^{2}$ C. Son and B. Ziaie, Appl. Phys. Lett. 92, 013509 (2008).

${ }^{3}$ M. W. Kowarz, U.S. Patent No. 6844960 B2 (18 June 2005).

${ }^{4}$ B. Legrand, A. S. Rollier, D. Collard, and L. Buchaillot, Appl. Phys. Lett. 88, 034105 (2006).

${ }^{5}$ A. Gooray, G. Roller, P. Galambos, K. Zavadil, R. Givler, F. Peter, and J. Crowley, J. Imaging Sci. Tech. 46, 415 (2002).

${ }^{6}$ T. L. Sounart, T. A. Michalske, and K. R. Zavadil, J. Microelectromech. Syst. 14, 125 (2005).

${ }^{7}$ H. V. Panchawagh, T. L. Sounart, and R. L. Mahajan, J. Microelectromech. Syst. 18, 1105 (2009).

${ }^{8}$ H. Zhang, M. S. Marma, E. S. Kim, C. E. McKenna, and M. E. Thompson, J. Micromech. Microeng. 15, 1911 (2005).

${ }^{9}$ T. Adrega, V. Chu, and J. P. Conde, J. Appl. Phys. 101, 094308 (2007).

${ }^{10}$ N. Scuor, P. Gallina, H. V. Panchawagh, R. L. Mahajan, O. Sbaizero, and V. Sergo, Biomed. Microdevices 8, 239 (2006).

${ }^{11}$ H. V. Panchawagh, F. F. Faheem, C. F. Herrmann, D. B. Serrell, D. S Finch, and R. L. Mahajan, Sens. Actuators A: Phys. 134, 11 (2007).

${ }^{12}$ D. Sameoto, T. Hubbard, and M. Kujath, J. Micromech. Microeng. 14, 1359 (2004).

${ }^{13}$ See supplementary material at http://dx.doi.org/10.1063/1.3389491 for a magnified view of the partial stability region and $V_{\mathrm{ns}}$ versus $X$ curves at selected values of $\rho$. 